\title{
An enhanced resolution brightness temperature product for future conical scanning microwave radiometers
}

\author{
Ferdinando Nunziata, Senior Member, IEEE, Matteo Alparone, Student Member, IEEE, \\ Adriano Camps, Fellow Member, IEEE, Hyuk Park, Senior Member, IEEE, Alberto M. Zurita \\ Claudio Estatico, and Maurizio Migliaccio, Fellow Member, IEEE
}

\begin{abstract}
An enhanced spatial resolution brightness temperature product is proposed for future conical scan microwave radiometers. The technique is developed for Copernicus Imaging Microwave Radiometer (CIMR) measurements that are simulated using the CIMR antenna pattern at L-band, and the measurement geometry proposed in the Phase A study led by AIRBUS. An inverse antenna pattern reconstruction method is proposed. Reconstructions are obtained using two CIMR configurations, namely using measurements collected at L-band by the forward (FWD) scans only, and combining forward and backward (FWD+BWD) scans. Two spatial grids are adopted, namely $3 \mathrm{~km} \times 3 \mathrm{~km}$ and $36 \mathrm{~km} \times 36 \mathrm{~km}$. Simulation results, referred to synthetic and realistic reference brightness fields, demonstrate the soundness of the proposed scheme that provides brightness temperature fields reconstructed at a spatial resolution up to $\sim 1.9$ times finer than the measured field when using the FWD+BWD combination.
\end{abstract}

Index Terms-Resolution enhancement, inverse problem, microwave radiometer, multi-channel data fusion.

\section{INTRODUCTION}

The Copernicus Imaging Microwave Radiometer (CIMR) is one of the six high-priority missions within the European Copernicus Expansion program. CIMR will be a conical- scanning multi-frequency microwave radiometer, that will support the Integrated EU policy for the Arctic and Copernicus needs by providing observations of sea-surface temperature, sea-ice concentration and sea-surface salinity with a focus on high latitude regions [1]. Although most of these ocean and sea

M. Alparone, F. Nunziata and are with the Dipar- timento di Ingegneria, Università degli Studi di Napoli Parthenope, 80143 Napoli, Italy (e-mail: matteo.alparone@uniparthenope.it; ferdi- nando.nunziata@uniparthenope.it)

M. Migliaccio is with the Dipartimento di Ingegneria, Università degli Studi di Napoli Parthenope 80143 Napoli, Italy, and also with the Istituto Nazionale di Geofisica e Vulcanologia, 00143 Rome, Italy (e-mail: mnm.migliaccio@gmail.com)

A. Camps is with Unidad María de Maetzu, CommSensLab-UPC, Dept. of Signal Theory and Communications, Universitat Politenica de Catalunya BarcelonaTech and Politécnica Institut d'Estudis Espacials de Catalunya IC/UP, Barcelona, Spain

Hyuk Park is with CommSensLab, Unidad María de Maetzu, Department of Signal Theory and Communications, IEEC/CTE-UPC, Universitat Politécnica de Catalunya 08034, Barcelona, Spain, and also with the Department of Physics, Castelldefels School of Telecommunications and Aerospace Engineering, UPC- BarcelonaTech 08034, Barcelona, Spain

A. M. Zurita is with AIRBUS Defence Space, Space Systems, Madrid, Spain

C. Estatico is with the Dipartimento di Matematica, Università degli Studi di Genova, 16146 Genova, Italy (e-mail: estatico@dima.unige.it). The work of Claudio Estatico is partially supported by GNCS-INDAM, Italy
TABLE I

CIMR CHANNELS SPATIAL RESOLUTION AND RADIOMETRIC SENSITIVITY

\begin{tabular}{lll}
\hline Frequency $(\mathrm{GHz})$ & Spatial Resolution $(\mathrm{km})$ & $\mathrm{Ne} \Delta \mathrm{T}[\mathrm{K}]$ \\
\hline 1.414 & 60 & 0.3 \\
6.925 & 15 & 0.2 \\
10.65 & 15 & 0.3 \\
18.6 & 5 & 0.4 \\
36.5 & 5 & 0.7 \\
\hline
\end{tabular}

ice variables can be estimated using global-scale visible and infrared remotely sensed measurements, the temporal sampling is severely limited by weather conditions, which are specially challenging in polar regions. Hence, passive measurements provided by microwave radiometers are required as they allow to estimate ocean and sea ice variables even under cloudy weather conditions.

State-of-the-art operational microwave radiometers provide measurements of poor spatial resolution, in particular at the lowest frequency channels, and this limits their operational use in coastal and marginal ice zones. CIMR is designed to provide measurements with an increased accuracy and spatial resolution than the state-of-the-art ones. It will be equipped with a deployable mesh reflector with a $7.4 \mathrm{~m}$ diameter that provides measurements over $\mathrm{a} \sim 1900 \mathrm{~km}$ swath using both $\mathrm{V}$ - and $\mathrm{H}$-polarized channels at $\mathrm{L}, \mathrm{C}, \mathrm{X}, \mathrm{Ku}$, and $\mathrm{Ka}$ bands, with a Noise Equivalent Delta Temperature $(\mathrm{Ne} \Delta \mathrm{T})$ ranging from 0.2 to $0.7 \mathrm{~K}$ (Table I). CIMR mission will fly at $830 \mathrm{~km}$ altitude in a dawn-dusk Sun-synchronous orbit designed to guarantee $95 \%$ global coverage every day with one satellite with sub-daily coverage over the polar regions. It will spin at around $8.2 \mathrm{rpm}$ with an on-board zenith angle (OZA) of $55^{\circ}$. CIMR is designed to perform measurements over both a forward scan (FWD), and a backward scan arcs (BWD) with a time lag between FWD and BWD scans of $260 \mathrm{~s}$ (Fig. 1). The footprint size of each CIMR frequency channel is limited by the size of the mesh reflector and the measurement integration time, see Table I.

CIMR will provide both un-gridded and gridded products. The former preserves the native scan geometry; while the latter consists of CIMR measurements projected onto an image grid. This study focuses on the generation of gridded products. Several approaches have been proposed in the literature to deal 


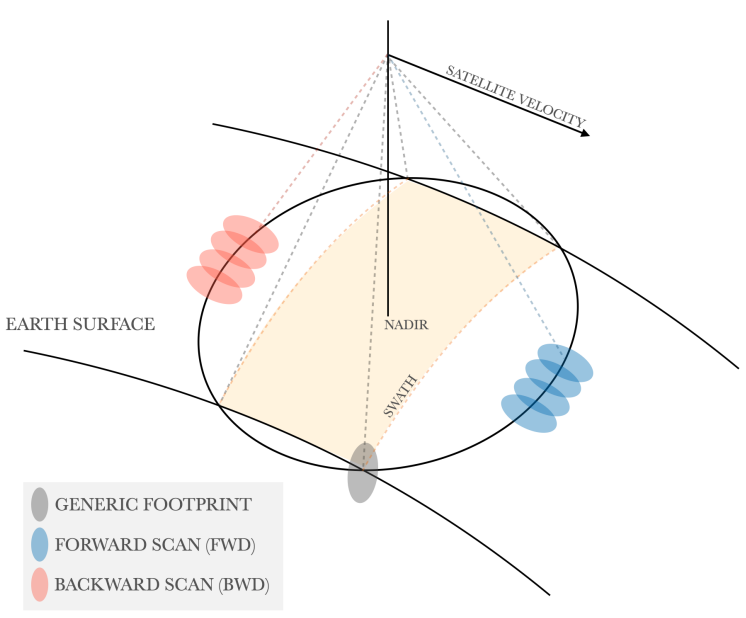

Fig. 1. Schematic overview of the CIMR measurement geometry. Adapted from CIMR Mission Requirement Document [2]

with the generation of gridded products starting from swathbased radiometer or scatterometer measurements that call for a trade-off among noise, spatial and temporal resolutions. The simplest approach consists of the so-called "drop-inthe-bucket" method that is also known as gridding (GRD) algorithm. This method, which results in a low-noise and low-resolution product, is the straightforward approach to obtain gridded products [3]. An alternative choice consists of using image reconstruction methods that result in finer spatial resolution gridded products, although at the expense of some noise amplification. These enhanced spatial resolution products are of paramount importance especially when dealing with passive microwave radiometer applications related to coastal areas and when the measurements collected by the different microwave radiometer channels have to be combined to provide reliable estimations of the geophysical parameter of interest. This is the case, for instance, of sea ice concentration estimation [4], [5]. Several image reconstruction methods have been proposed in the literature to estimate the brightness temperature field on a finer spatial resolution grid that consist of solving a linear underdetermined ill-conditioned system of equations. In the context of the NASA-sponsored MEaSUREs program, finer spatial resolution products are obtained using the well known Backus-Gilbert (BG) method [6]-[11] and an improved version of the scatterometer image reconstruction method (SIR) specialised to the radiometer case (rSIR) [10], [11]. In addition to these methods, other approaches have been proposed consisting of exploiting linear algebra tools to deal with the ill-posedness of the inverse problem. They typically rely on the 2 -norm to constrain the sought solution [12][15]. In [16]-[19] iterative regularizing methods to enhance the spatial resolution of microwave radiometer measurements are designed where constraints on the sought solution are imposed both in the Hilbert and Banach spaces. Recently, an iterative image reconstruction algorithm that jointly exploits lowerand higher-frequency channels of a multichannel microwave radiometer is proposed and verified over both simulated and actual Special Sensor Microwave Imager (SSM/I) measurements [20]. In [21] a method that relies on the combination of measurements collected by the lower and higher-frequency channels of a multi-frequency radiometer was proposed. The latter is based on the Smoothing Filter-based Image Modulation technique (SFIM) and it has been used in [22] for an intercomparison between remotely sensed soil moisture product . In [23], [24] an enhanced Soil Moisture and Ocean Salinity (SMOS) brightness temperature is obtained using a number of deconvolution algorithms showing that a product, whose spatial resolution can be enhanced by a factor 1.77 , can be achieved. In [25] the enhancement of the MWRI microwave radiometer measurements collected by the Chinese FY-3C mission is addressed using a deep learning (DL) method.

In this study, L-band CIMR-like measurements computed from realistic reference temperature brightness fields are simulated using the actual L-band CIMR antenna pattern and the actual CIMR measurement geometry. Reconstructions at enhanced spatial resolution are obtained using a gradient-like iterative regularization method with constraints in the Hilbert space. Two enhanced resolution spatial grids, namely $3 \times 3$ $\mathrm{km}$ and $36 \times 36 \mathrm{~km}$, are used to mimic the two measurements configurations. The latter consists of exploiting noisy CIMR measurements collected using the FWD scans only or combining FWD+BWD [11], [26], [27]. Numerical results show that an enhanced spatial resolution brightness temperature product can be achieved starting from measurements obtained by a system that mimics the actual CIMR measurement geometry. The rest of the paper is organised as follows: in Section II the theoretical background is briefly reviewed. In Section III the experimental setup is described and some meaningful showcases are presented and discussed both quantitatively and quantitatively. The concluding remarks are drawn in Section IV.

\section{THEORETICAL BACKGROUND}

The Fredholm integral equation that links (neglecting atmospheric effects) the CIMR radiometer measurement at a given channel with the brightness temperature can be written as [10]:

$$
T_{A}=\bar{G}^{-1} \iint_{\Omega} G(s) T_{B}(\mathbf{s}) d s
$$

where $\bar{G} \equiv \iint_{\Omega} G(s) d s$ is the antenna solid angle, being $G(\cdot)$ the smooth integral kernel that depends on the antenna gain pattern, $T_{A}(\cdot)$ stands for the measured antenna temperature, and $T_{B}(\cdot)$ is the unknown brightness temperature field to be reconstructed. The integration variable $s=(\gamma, \theta)$ contains the azimuth $(\gamma)$ and elevation angles $(\theta)[10]$. The linear direct problem described by (1) can be cast in a discrete setting in the form [16], [18], [19]:

$$
A \mathbf{x}=\mathbf{y}
$$

where the matrix $A$ contains the sampling of the CIMR projected antenna pattern, the $\mathbf{x} \in \mathbb{R}^{m}$ vector consists of the brightness temperature $T_{B}$ to be retrieved, and $\mathbf{y} \in \mathbb{R}^{n}$ is the vector of noisy measurements. The system (2) is illconditioned, since it comes from an ill-posed continuous linear 
problem, and it is also underdetermined, since the unknown $T_{B}$ has to be retrieved on a finer spatial resolution grid, i.e. $n>m$. This implies that constraints on the sought solution must be imposed to deal with the underdetermined nature of the problem; while regularising schemes must be adopted to mitigate the noise amplification when inverting the system (2). In this study, the discretised problem (2) is inverted by minimizing the 2-norm of the following residual:

$$
\Omega_{2}=\frac{1}{2}\|A \mathbf{x}-\mathbf{b}\|_{2}^{2}=\frac{1}{2} \sum_{i=1}^{n}(A \mathbf{x}-\mathbf{b})_{i}^{2} .
$$

This task can be accomplished using a gradient-like regularisation scheme, i.e., an iterative method where the number of iterations plays the role of regularisation parameter [28]. The generic $k+1$ iteration is given by [17]:

$$
\mathbf{x}_{k+1}=\mathbf{x}_{k}+\lambda_{k} \mathbf{p}_{k}
$$

where $\mathbf{x}_{0}$ stands for the initial guess and $\mathbf{x}_{k}$ for the $k$-th iteration, $\lambda_{k}>0$ is the step-size, and $\mathbf{p}_{k}$ is a descent direction at the point $\mathbf{x}_{k}$ for $\Omega_{2}$. In this study $\mathbf{p}_{k}=-\nabla \Omega_{2}\left(\mathbf{x}_{k}\right)$ is used. The latter is at the basis of the Landweber and steepest descent methods [29]. Since $\nabla \Omega_{2}\left(\mathbf{x}_{k}\right)=A^{*}\left(A \mathbf{x}_{k}-\mathbf{b}\right)$, the $\mathbf{x}_{k+1}$ iteration (4) becomes:

$$
\mathbf{x}_{k+1}=\mathbf{x}_{k}-\lambda A^{*}\left(A \mathbf{x}_{k}-\mathbf{b}\right)
$$

where $A^{*}$ denotes the adjoint operator of $A$. It can be demonstrated that an optimal choice for the step-size $\lambda$ is given by [16]:

$$
\lambda_{k}=\lambda=\frac{1}{\left\|A^{*} A\right\|}
$$

To find the regularising parameter $k$, i.e., the number of iterations to be performed to achieve an accurate enough reconstruction, the discrepancy principle is used [26]. It consists of choosing the smallest iteration number $k$ such that:

$$
\left\|A \mathbf{x}_{k}-\mathbf{b}\right\| \leq \varepsilon
$$

where $\varepsilon$ is an estimate of the 2-norm of the error, i.e., $\left\|n^{2}\right\|=\varepsilon$ and $c \geq 1$ rules the tradeoff between regularization and noise amplification [18].

\section{NUMERICAL EXPERIMENTS}

In this section the methodology to simulate CIMR-like measurements is first described; then, the objective metrics to discuss the enhanced spatial resolution product are discussed and finally, some meaningful numerical experiments are presented and discussed.

\section{A. Simulated CIMR measurements}

The forward model (2) is implemented according to the block diagram depicted in Fig. 2. Three reference fields are discussed, see Fig. $3 a$ and $3 b-3 c$ that refer to a synthetic scene and a realistic one, respectively. The synthetic scene (Fig. 3a) is used to estimate the pixel response function, i.e, the impulse response function that includes the effect of the reconstruction, of a given pixel. Hence, it provides information on the effective resolution of the image reconstruction algorithm. In this study, we follow the guidelines provided in [11] and we simulated a synthetic truth image that consists of $1 \times 1$ pixel Kronecker Delta Function whose $T_{B}$ is $10^{6} \mathrm{~K}$, located in the middle of the observed scene. The realistic reference field (Fig. 3b-3c) consists of an actual Advanced Microwave Scanning Radiometer 2 (AMSR2) Ka-band scene collected over the Western part of the Mediterranean sea that includes part of Italy, France, Spain and North African countries. The scene is obtained averaging AMRS-2 data over 1 month to reduce noise, and the image dynamics is adjusted to match Lband microwave radiometer measurements. The native spatial resolution is downgraded to $36 \times 36 \mathrm{~km}$, see Fig. $3 \mathrm{~b}$, and $3 \times 3 \mathrm{~km}$, see Fig. $3 \mathrm{c}$, for the purposes of this study. Note that, to reduce the size of the scene interpolated over the $3 \times 3 \mathrm{~km}$ spatial grid, only the central part of Fig. $3 \mathrm{~b}$ is considered to generate the reference field of Fig. 3c.

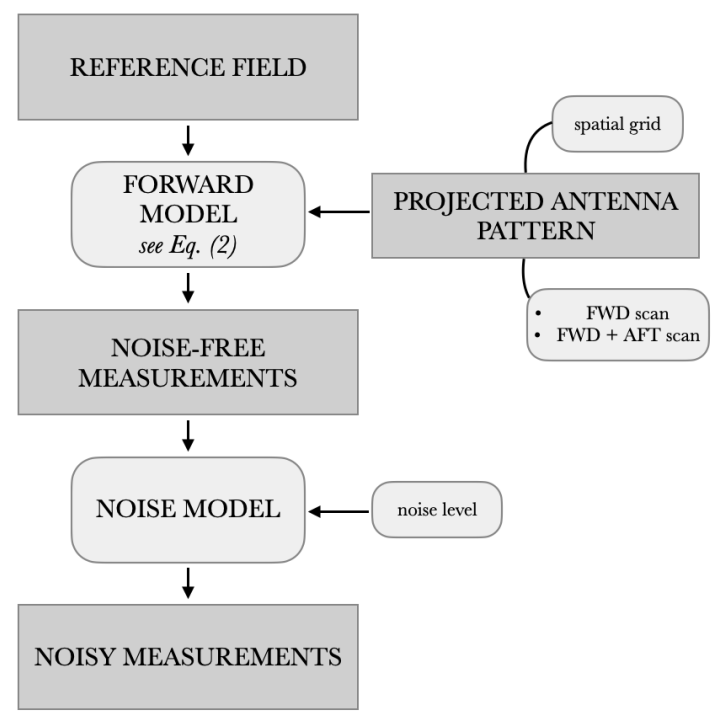

Fig. 2. Block diagram to depict the generation of noisy CIMR measurements onto the CIMR native spatial grid.

The system matrix $A$ in eq. (2) contains the sampled antenna pattern at the grid points of the reference field $\mathbf{x}$, i.e., $A=\left(\mathbf{A}_{1}, \mathbf{A}_{2}, \ldots, \mathbf{A}_{m}\right)^{T}$ with $A_{i} \in \mathbb{R}^{m}$. In this study, the L-Band CIMR antenna pattern was provided by AIRBUS and it was simulated using GRASP antenna reference software [30], considering realistic feed horn patterns, reflector optics and mesh characteristics. The 2D antenna pattern in ground coordinates is depicted in Fig. 4a. At L-Band, the mesh characteristics and the reflector illumination give a smooth pattern. With a native resolution of $74 \times 45 \mathrm{~km}$, it scans the Earth with an across-scan and along-scan overlap of roughly $34 \%$ and $67 \%$, respectively (Fig. 4b). While the first is dictated by geometry and rotation speed, the second is controlled, in addition, by the sampling time, which can be adjusted to balance the overlap and noise. In this case, 4 samples per integration time are considered.

To analyse the reconstruction performance with respect 
to the level of oversampling, two measurement set-ups are considered: (a) measurements collected by FWD scans only (see blue dots in Fig. 5), (b) measurements collected by both FWD and BWD scans over the same area (see red and blue dots in Fig. 5).

Noisy measurements are generated considering a realistic noise upper bound of $1 \mathrm{~K}$ (see Table I). The latter is a bit larger than the expected CIMR one (see [2]) and, therefore, it represents a worst case to test the reconstruction performance. For each experiment, simulated CIMR measurements are linearly interpolated onto the finer spatial resolution grid to discuss the performance of image reconstruction against the straightforward linear interpolation. Simulated CIMR measurements in their native spatial grid are not shown to save space.

\section{B. Enhanced resolution product and performance analysis}

The enhanced spatial resolution product is obtained from the noisy simulated CIMR measurements discussed above using the gradient-like regularization scheme summarised in Section II. The reconstruction's performance is quantitatively analysed using the following objective metrics:

- Improvement Factor (IF): It provides quantitative information on the ability to reconstruct spot-like discontinuities. It is the contrast of the reconstructed, and the measured brightness fields of an isolated target:

$$
I F=\frac{b m_{r f}}{b m_{r e c}}
$$

where:

$$
b m=\sqrt{\frac{\text { area of the binary mask }}{\pi}}
$$

is obtained by evaluating the $-3 \mathrm{~dB}$ area of the reference $\left(b m_{r f}\right)$ and reconstructed $\left(b m_{r e c}\right)$ brightness temperature fields. The larger is IF, the larger is the improvement of the spatial resolution. IF approaching unity means that the brightness field is reconstructed at a spatial resolution similar to the measured one, i.e., the improvement of the spatial resolution is negligible.

- The minor $(a)$ and the major $(b)$ axes of the ellipse that best fits the $-3 \mathrm{~dB}$ contour of the spot-like discontinuity in the measured and reconstructed fields.

- Sharpening Factor (SF): It provides quantitative information on the ability to reconstruct abrupt discontinuities:

$$
S F=\frac{\gamma_{m}}{\gamma_{r e c}}
$$

where $\gamma$ is an angle defined in Fig. 6 to provide information on the broadening of the measured $\left(\gamma_{m}\right)$ and the reconstructed $\left(\gamma_{r}\right)$ fields with respect to the reference one. The larger is SF, the better is the sharpening of the reconstructed field with respect to the measurements.

- The point-wise correlation (CO) between the reference and the reconstructed fields.

- The normalised distance (ND) between the reconstructed and the reference fields:

$$
N D=\frac{\left\|\mathbf{x}_{\text {rec }}-\mathbf{x}_{\text {ref }}\right\|_{\text {Fro }}}{\left\|\mathbf{x}_{\text {ref }}\right\|_{\text {Fro }}}
$$
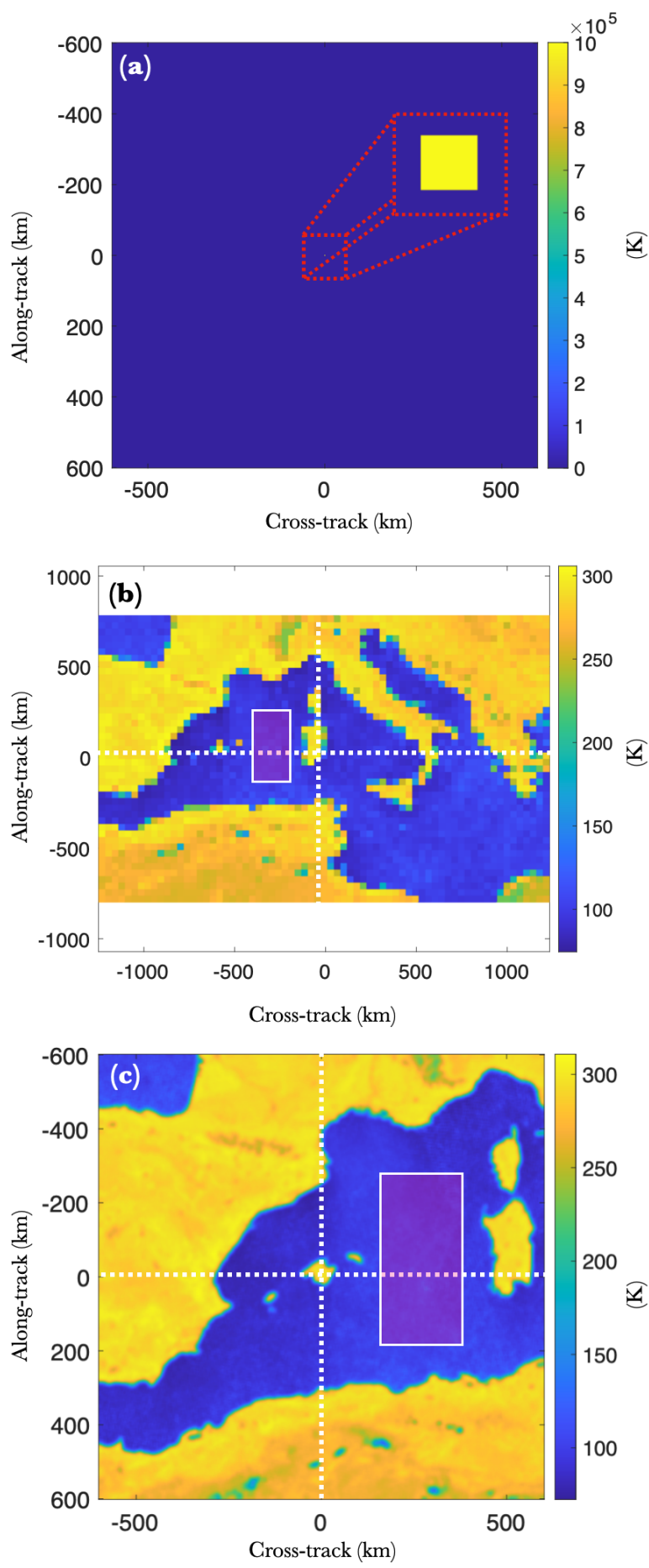

Fig. 3. Reference fields considered in this study: (a) $1 \times 1$ pixel Kronecker Delta Function located in the middle of the observed scene. An enlarged version of the hot spot is also annotated in the figure. Realistic reference field obtained averaging AMSR-2 brightness fields and downgraded to a $36 \times$ $36 \mathrm{~km} \mathrm{(b)} \mathrm{and} 3 \times 3 \mathrm{~km}$ (c) spatial resolution. 

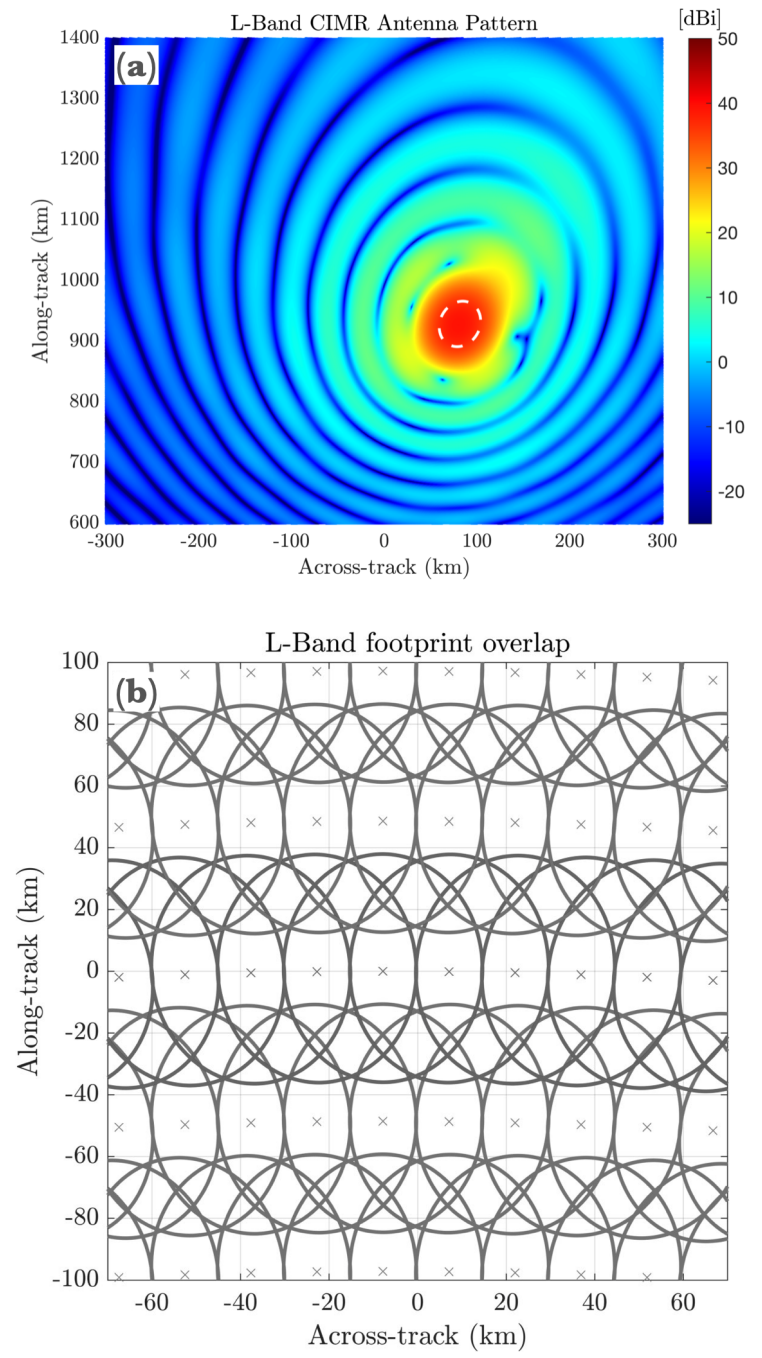

Fig. 4. L-Band CIMR antenna pattern and across-scan and along-scan overlapping, simulated using GRASP antenna reference software. (a) 2D LBand pattern projected on-ground at $0^{\circ}$ scan angle for the nominal CIMR altitude. This assumes that the subsatellite point is located at $(0,0)$ (alongtrack, across-track). The dashed white line marks the $3 \mathrm{~dB}$ contour from the peak directivity; (b) $3 \mathrm{~dB}$ contour lines on-ground with the sampling pattern resulting from the rotation speed, the satellite movement and the sampling time.

where $\|\cdot\|_{F r o}$ is the Froebenius norm, and $\mathbf{x}_{r e c}\left(\mathbf{x}_{r e f}\right)$ is the reconstructed (reference) brightness temperature field.

\section{Numerical Experiments}

The first experiment refers to the reconstruction of the spotlike discontinuity of Fig. 3a to analyse the performance in terms of the point spreading function. Note that the latter can vary according to the selected pixel; hence, we refer to a pixel located in the center of the swath [11]. Noisy measurements obtained by the forward model of Fig. 2 are depicted in Fig. $7 \mathrm{a}$ when using FWD scans only, and in Fig. 7b when using the FWD+BWD case. Following the guidelines provided in [11], for visualization purposes, the measured brightness field is linearly interpolated onto the finer spatial resolution grid and the brightness temperature is normalised to the maximum

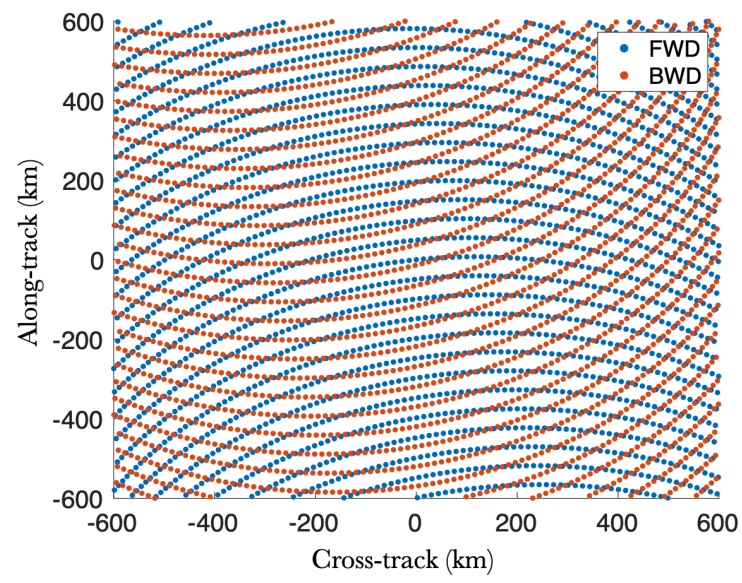

Fig. 5. Boresight position of each footprint related to the along-scan measurements depicted for a number of FWD (blue circles) and BWD scans (red circles).

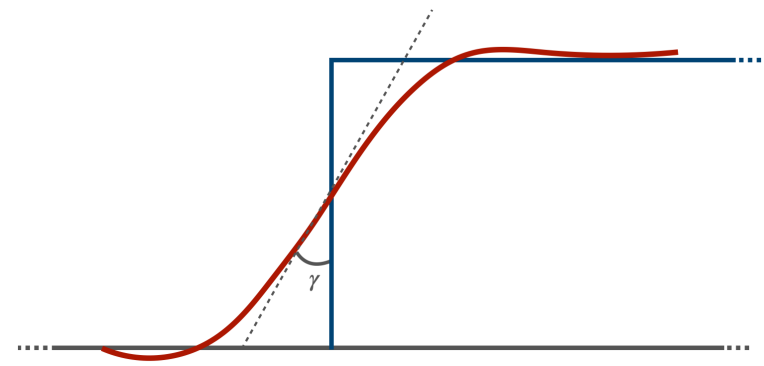

Fig. 6. Pictorial sketch of the angle $\gamma$ used to define the SF metric. The red curve stands for a measured/reconstructed profile; while the blue one is related to the reference profile.

value and depicted according to a decibel (dB) scale to visually inspect the $-3 \mathrm{~dB}$ size of the measured hot spot. As expected, the radiometer measurement process results in a smoothed hot spot. However, by visually contrasting Fig. $7 \mathrm{a}$ and $7 \mathrm{~b}$ one can note that the FWD+BWD combination results in a measured hot spot calling for sharper edges and lower over-smoothing. This can be quantitatively confirmed by the metrics $a$ and $b$ together with their geometric average listed in Table II. The reconstructed field at enhanced spatial resolution is depicted in Fig. $7 \mathrm{c}$ and $7 \mathrm{~d}$ when using FWD only, and in the FWD+BWD cases, respectively. The reconstructed field is sharper than the measured one in both the FWD and the FWD+BWD cases, although fluctuations due to the Gibbs phenomena apply in the area nearby the hot spot. To quantitatively discuss the performance of the reconstruction, quantitative metrics are evaluated, see Table II. The quantitative parameters confirm that a sharper field is reconstructed in both the along- and the across-scan directions. The best performance is achieved by the FWD+BWD combination that benefits from the increased overlapping among adjacent beams resulting form the combination of FWD and BWD scans, and provides a sharper reconstructed field. This behaviour is also confirmed by the IF metric (8) that is equal to 1.68 and 1.49 for the FWD+BWD, and the FWD cases, respectively. Hence, the reconstruction method yields an effective spatial resolution that is finer than 
TABLE II

PERFORMANCE ANALYSIS RELATED TO THE REFERENCE FIELD OF FIG. 3A. NOTE THAT “AVERAGE” STANDS FOR THE GEOMETRIC AVERAGE OF THE $a$ AND $b$ PARAMETERS DEFINED IN SECTION III-B

\begin{tabular}{clll}
\hline & & Measured Field $(\mathrm{km})$ & Reconstructed Field $(\mathrm{km})$ \\
\hline \multirow{4}{*}{ FWD } & $a$ & 55 & 32 \\
& $b$ & 83 & 64 \\
& average & 67.5 & 42.2 \\
& IF & & 1.49 \\
\hline \multirow{5}{*}{ FWD+BWD } & $a$ & 45 & 24 \\
& $b$ & 72 & 48 \\
& average & 56.9 & 33.9 \\
& IF & & 1.68 \\
\hline
\end{tabular}

the measurement one.

The second experiment is related to the reference field depicted in Fig. 3b. The noisy measurements, linearly interpolated onto the reference field grid, are depicted in Fig. 8a and $8 \mathrm{~b}$ when using FWD scans only, and in the FWD+BWD case, respectively. The measured brightness field is smoothed by the radiometer measurement process, and this results in a smoother land/sea transition that makes the coastlines very blurred. In addition, the islands of Sardinia and Corsica are not well-separated as well as the Sicily island, that appears linked to the Italian peninsula. Some of the islands of the Balearic archipelago can be hardly recognised. Negligible differences can be observed by visually contrasting the measured fields of Fig. 8a and 8b. This implies that, at this spatial grid, the joint exploitation of FWD and BWD scans does not result in improved performance in the linearly interpolated gridded measurements. The reconstructed field at enhanced spatial resolution is depicted in Fig. 8c and 8d for the FWD scans and the FWD+BWD scans combination, respectively. It can be noted that sharper edges and coastlines are observed in both the reconstructions and, in addition, the Balearic islands (on the left-hand side of the image) are better delineated and separated. The same applies for the separation between Sardinia and Corsica islands, and the separation between Sicily island and the Italian peninsula that improves with respect to the measured field. Note that Gibbs' oscillations that have appeared are inherently related to the minimization of the energy norm (3). By visually contrasting the reconstructed fields of Fig. 8c and 8d one can note that the joint exploitation of FWD+BWD scans reduces Gibbs' related fluctuations. This is due to the larger overlapping among the projected beams (Fig. 5) that, at once, results in a larger redundancy in the measurements. To provide a deeper analysis of the reconstruction's performance, brightness temperature profiles evaluated along with the horizontal (a) and vertical (b) transects (Fig. 3b) kept over the reference, measured and reconstructed fields are depicted in Fig. 9. The brightness temperatures profiles related to the horizontal transect of Fig. 3b are depicted in Fig. 9a for both the FWD and FWD+BWD cases. The two panels share the same legend depicted in Fig. 9b. The linearly interpolated measured profiles resulting from the FWD and FWD+BWD cases do not exhibit remarkable differences. The reconstructed profile at enhanced spatial resolution is sharper than the measurements in both the FWD and FWD+BWD
TABLE III

OBJECTIVE METRICS RELATED TO THE PROCESSING OF THE REFERENCE FIELD OF FIG. 3B. THE METRIC SF IS EVALUATED AT THE ABRUPT DISCONTINUITIES MARKED AS "P1" AND "P2" IN FIG. 9

\begin{tabular}{cllll}
\hline & CO & ND & SF (P1) & SF (P2) \\
\hline FWD & 0.9941 & 0.0497 & 2.74 & 1.44 \\
FWD+BWD & 0.9983 & 0.0267 & 3.95 & 2.68 \\
\hline
\end{tabular}

cases, and it better fits the reference profile. The FWD+BWD combination results in a reconstructed profile slightly better than the FWD one in terms of both sharper edges and reduced Gibbs' fluctuations. The vertical transect (Fig. 9b) shows that the linearly interpolated measured profile related to the FWD+BWD combination better fits the reference profile with respect to the FWD scans only, specially at abrupt discontinuities. A notable example is related to the separation between the islands of Sardinia and Corsica, see the light blue ellipse, labelled as "P1" in Fig. 9b, which is better reconstructed using the FWD+BWD combination. The same applies for the land/sea transition, see the reddish ellipse labelled as "P2" in Fig. 9b. In addition, the FWD+BWD combination results in lower Gibbs' fluctuations. To quantitatively discuss the reconstruction's performance the figures of merit $\mathrm{CO}, \mathrm{ND}$ and SF are listed in Table III. The CO and ND metrics are evaluated over the whole image, while the SF metric is evaluated considering the abrupt discontinuities enclosed in the ellipses labeled as "P1" and "P2" in Fig. 9. The figures of merit confirm that the FWD+BWD combination always provides the best reconstruction performance. Note that the IF metric (Eq. (8)) is not evaluated, since the observed scene is such that the $36 \mathrm{~km}$ grid does not offer hot spots calling for a brightness temperature large enough to evaluate IF.

The third experiment is related to the reference field depicted in Fig. 3c, and it aims at discussing the performance of the enhanced product using a $3 \mathrm{~km} \times 3 \mathrm{~km}$ spatial grid. The measured noisy fields, obtained using FWD scans and the FWD+BWD combination, and linearly interpolated onto the $3 \mathrm{~km} \times 3 \mathrm{~km}$ grid, are depicted in Fig. 10a and 10b, respectively. Even in this case, the radiometer measurement process makes edges and land/sea transitions very blurred even in the interpolated image and makes the Balearic islands hardly recognizable. Negligible differences can be observed by contrasting Fig. 10a and 10b. The measured field resulting from the FWD+BWD combination results in sharper edges, and better delineated Balearic islands, if compared to the resulting field from FWD scans only. However, the latter calls for a better separation between Sardinia and Corsica islands. The field reconstructed at enhanced spatial resolution is depicted in Fig. 10c and 10d for the FWD and FWD+BWD combination, respectively. By visually inspecting reconstructions one can note that sharper edges and better defined coastlines are achieved. The larger islands (Sardinia and Corsica) are better separated, and the Balearic islands are better delineated. By visually inspecting reconstructions related to FWD only, and FWD+BWD combination one can note that, even in this case, the latter results in the best performance, see for instance the better separation between Sardinia and 

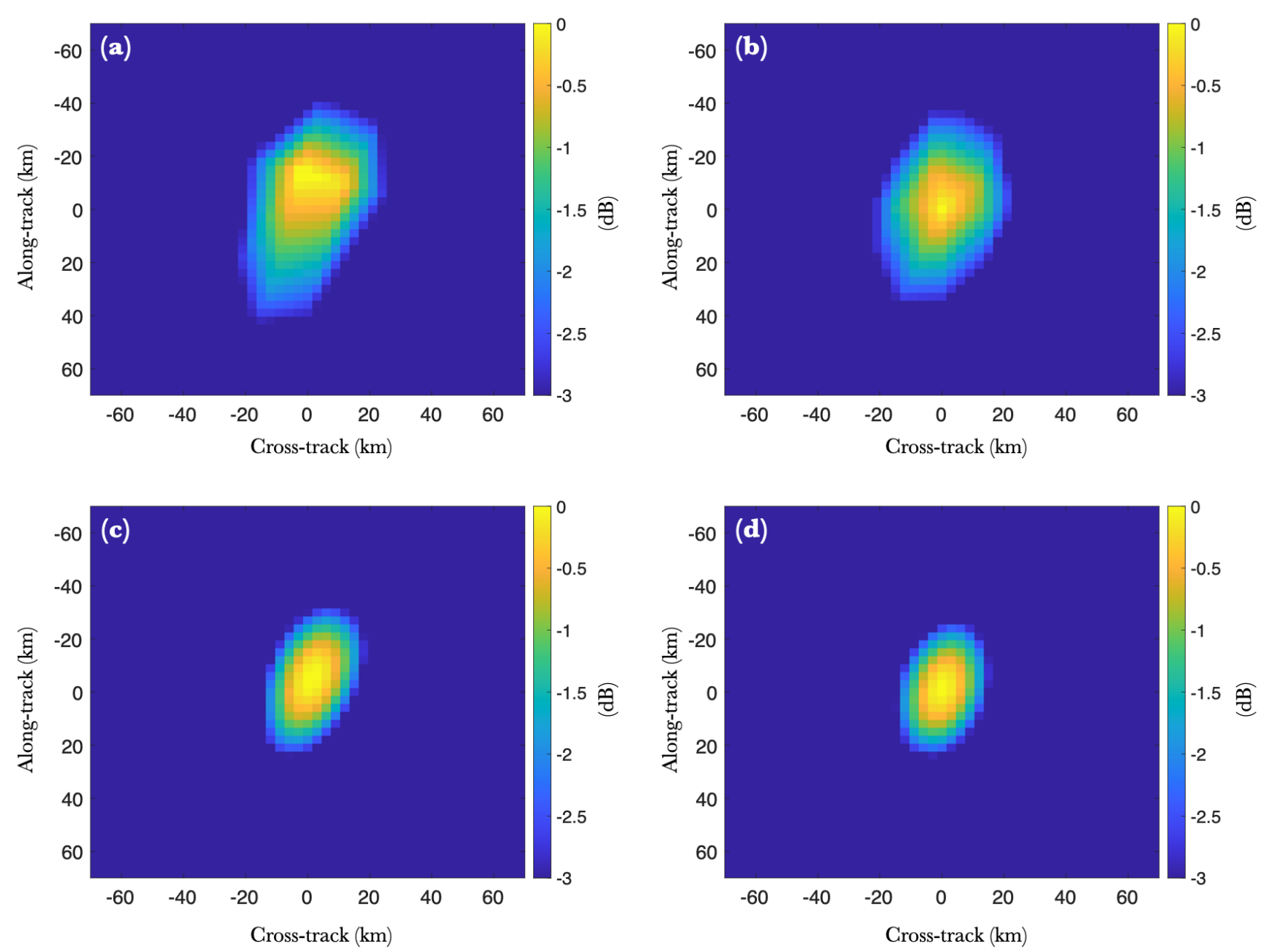

Fig. 7. Noisy CIMR measurements linearly interpolated onto the finer spatial grid (first row) and reconstructed field at enhanced spatial resolution (second row) related to the reference field of Fig. 3a and obtained using FWD scans only (first column) and using the FWD+BWD combination (second column). Note that, since $T_{B}$ values larger than $-3 \mathrm{~dB}$ are shown, side lobes are not visible in the above images.

Corsica and the stronger signal resulting from the Balearic islands. To provide a deeper analysis of the reconstruction's performance, brightness temperature profiles evaluated along with the horizontal (a) and vertical (b) transects (Fig. 3c) kept over the reference, measured and reconstructed fields are depicted in Fig. 11. The brightness profiles related to the horizontal transect of Fig. 3c are depicted in Fig.11a for both the FWD and FWD+BWD cases. The two panels share the same legend depicted in Fig. 11b. The reconstructed profile at enhanced spatial resolution is sharper than the measurements and it better fits the reference profile, see e.g. the land/sea transition enclosed in the reddish ellipse labelled as "P" in Fig. 11a. The FWD+BWD combination results in a reconstructed profile slightly better than the FWD one in terms of reduced Gibbs' fluctuations over land, see e.g. the reconstruction of the Sardinia island in the right part of the plot. The vertical transect (Fig. 11b) shows that, again, the profile reconstructed at enhanced spatial resolution using FWD+BWD scans performs best in terms of reconstruction of abrupt discontinuities. A notable example related to both the horizontal and vertical transects is the Mallorca island, see the light blue ellipses, which is better reconstructed using the FWD+BWD combination. This behaviour is also confirmed by visually contrasting an enlarged version of the Mallorca and Menorca islands, see Fig. 12, where the $-3 \mathrm{~dB}$ binary masks associated with the fields measured using FWD (Fig. 12a) and FWD+BWD (Fig. 12c) are shown along with the reconstructed fields for the FWD (Fig. 12b) and FWD+BWD (Fig. 12d) cases. It can be noted that the two islands are not resolved by the CIMR measurement. These latter are separated in the enhanced spatial resolution product. In addition, the FWD+BWD combination provides a sharper and less oversmoothed reconstruction of the islands. To quantitatively discuss the performance of the reconstructions the figures of merit CO, ND, IF and SF are listed in Table IV. The metric IF is evaluated with respect to the Mallorca island, over the area enclosed in the boxes of Fig. 12. Note that for the CIMR-like measurements case the area of the Mallorca island has been evaluated considering the ideal border depicted as a dotted red line in Fig. 12a and Fig. 12c. The metric SF refers to the abrupt discontinuity enclosed in the ellipse labeled as "P" in Fig. 11a (coast of Valencia, Spain). All the figures of merit 

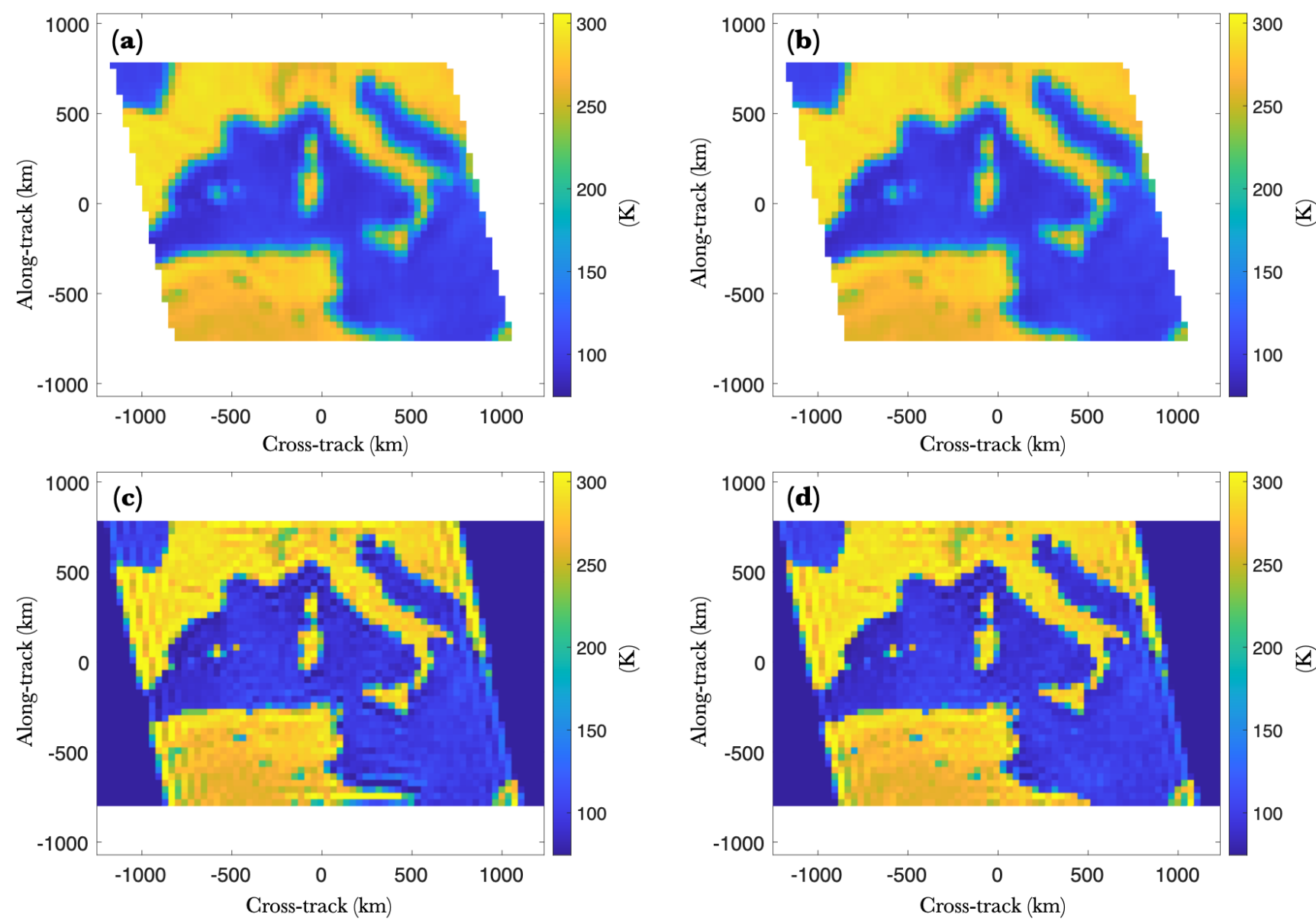

Fig. 8. Noisy CIMR measurements (linearly interpolated onto the finer spatial grid) related to the reference field of Fig. $3 \mathrm{~b}$ and obtained using FWD scans only (a) and the combination of FWD and FWD+BWD scans (b). Reconstructed fields at enhanced spatial resolution obtained using FWD scans only (c) and the combination of FWD and FWD+BWD scans (d).
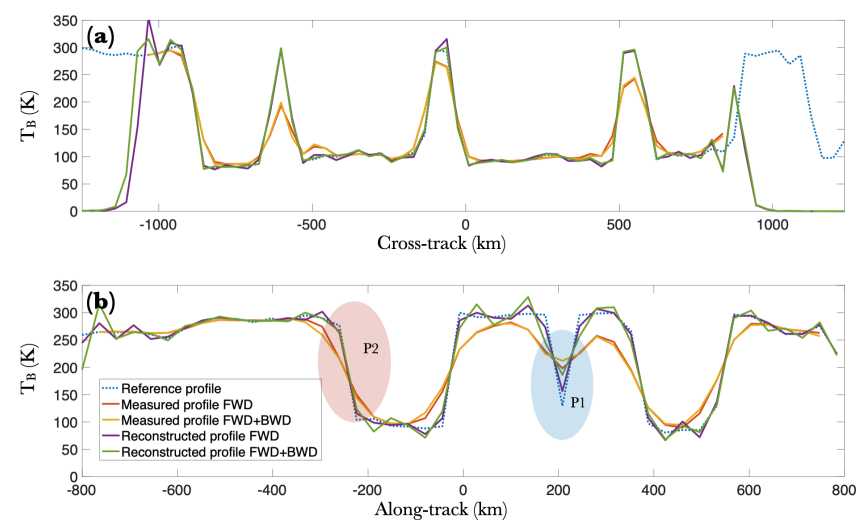

Fig. 9. Brightness temperature profiles related to the along-scan (a) and across-scan (b) transects depicted in Fig. $3 b$ evaluated using the field reconstructed at enhanced spatial resolution and the measured field obtained using the FWD only and the FWD+BWD combination. Note that the reference profile is also annotated.

show that the reconstruction performance improves when the FWD+BWD combination is used.
TABLE IV

OBJECTIVE METRICS TO DESCRIBE THE RECONSTRUCTION'S PERFORMANCE OF THE METHOD UNDER REALISTIC NOISY MEASUREMENTS RELATED TO THE REFERENCE FIELD OF FIG. 3C

\begin{tabular}{cllll}
\hline & CO & ND & SF (P1) & SF (P2) \\
\hline FWD & 0.8766 & 0.0188 & 1.57 & 1.21 \\
FWD+BWD & 0.9209 & 0.0142 & 1.89 & 1.42 \\
\hline
\end{tabular}

TABLE V

MEAN VALUe AND RMSE BETWEEN THE RECONSTRUCTED AND THE REFERENCE FIELDS FOR THE $36 \times 36$ KM AND THE $3 \times 3$ KM GRIDDED PRODUCTS

\begin{tabular}{ccc}
\hline & Mean Value $[\mathrm{K}]$ & RMSE $[\mathrm{K}]$ \\
\hline $36 \times 36 \mathrm{~km}$ & 0.07 & 1.7 \\
$3 \times 3 \mathrm{~km}$ & 0.03 & 2.6 \\
\hline
\end{tabular}

\section{Validation on real L-band measurements}

To test the performance of the proposed approach on actual L-band radiometer measurements we used the L-band conical scanning radiometer operated by the Soil Moisture Active Passive (SMAP), i.e., a real aperture radiometer that is very similar to the L-band CIMR configuration. The SMAP passive 
TABLE VI

ERROR STATISTICS FOR THE RECONSTRUCTION OF FIG. 5A IN [11] USING THE LW METHOD

\begin{tabular}{llll}
\hline & Mean & STD & RMS \\
\hline LW & -0.63 & 4.44 & 4.49 \\
\hline
\end{tabular}

sensor operates at L-band $(1.41 \mathrm{GHz})$ with a $24-\mathrm{MHz}$ bandwidth. It collects measurements of the horizontal $(\mathrm{H})$, vertical (V), and third and fourth Stokes parameter polarizations with a resolution of $40 \mathrm{~km}$ and a radiometric uncertainty of $1.3 \mathrm{~K}$ [31]. The SMAP scanning concept is similar to the CIMR one illustrated in Fig. 1, with a spin rate of $14.6 \mathrm{rpm}$ and a swath width of $100 \mathrm{~km}$. The fourth experiment is to reconstruct on a finer spatial resolution grid SMAP measurements acquired in 2021 [32] over an area that includes Cuba, Haiti and the Bahamas archipelago, see Fig. 13a where the observed area is depicted in the SMAP native spatial grid. Note that black pixels stand for gaps values that can occur under the conditions explained in the data User Guide [32]. As expected, blurred edges and coastlines appear in the native SMAP measurements field. The field at enhanced spatial resolution is depicted in Fig. $13 \mathrm{~b}$ where one can note better defined edges and coastlines.

\section{E. Noise analysis}

To fully discuss the performance of the enhanced resolution brightness product, the noise associated to the reconstructions is analysed in both the $36 \times 36 \mathrm{~km}$ and the $3 \times 3 \mathrm{~km}$ grids. The mean value and RMSE between the reconstructed and the reference fields are evaluated over a region of interest (ROI) that appears homogeneous in the reference fields, see reddish box in Fig. 3b and Fig. 3c. The ROI covers an area of roughly $70000 \mathrm{~km}^{2}$ extending between the Balearic islands and the island of Sardinia. The results of the noise amplification analysis are shown in Table V. It can be noted that reconstructions are almost unbiased in both the grids and, although noise amplifies with decreasing the grid size, the noise level is a bit smaller than the SMAP and CETB gridded products discussed using the same metric in [11].

\section{F. Comparison with previous methods}

To inter-compare the performance of the proposed approach with the state-of-the-art ones, we address the reconstruction of the synthetic reference field presented in Fig. 5a of [11]. First, the SMAP single-pass measured field is generated according to [11] and, then, the field is reconstructed at enhanced spatial resolution on the same $3.125 \mathrm{~km}$ grid used in [11] using single-pass measurements. Finally, the error statistics are evaluated and listed in Table VI. The proposed method results in reconstruction performance similar to the one listed in [11] (see Table III of [11]), which refers to the BG and rSIR algorithms. In particular, a bit better STD and RMS are achieved; while the mean error value worsens.

\section{CONCLUSIONS}

An enhanced spatial resolution brightness temperature product is developed for CIMR noisy measurements collected by future conical scanning microwave radiometer and tested using simulated L-band CIMR data and actual SMAP ones. CIMR measured fields are obtained using a realistic L-band CIMR antenna pattern and simulating the CIMR measurement geometry. The reconstruction of the field at enhanced spatial resolution is accomplished using an inverse antenna pattern gradient-like iterative method whose reconstruction's performance is discussed in a quantitative way through objective metrics. The main outcomes can be summarised as follows: a) the CIMR measurements can be successfully provided on finer resolution grids; $b$ ) the reconstructions always outperform simply gridded measurements in terms of sharpness and ability to correctly delineate hot spots; c) the combination of FWD and BWD results in reconstructions that always outperforms the ones that exploit FWD scans only; d) the reconstructed field is enhanced by a factor spanning from $\sim$ (FWD case) up to $\sim 1.9$ (FWD+BWD); e) the method works fine on actual L-band SMAP measurements and results in slightly improved performance in terms of CIMR noise amplification with respect to state-of-the-art ones.

\section{ACKNOWLEDGMENTS}

This work has been produced for the European Space Agency (ESA) in the frame of the Copernicus programme as a partnership between ESA and the European Commission. We thank AIRBUS Defence and Space, in the persons of Josep Closa Soteras and Alasdhair Beaton, for their assistance and support.

\section{REFERENCES}

[1] European Commission, EU Arctic Policy, available at $\{\mathrm{https}: / /$ eeas. europa.eu/arctic-policy/eu-arctic-policyen\}.

[2] CIMR Mission Science Division, CIMR Mission Requirements Document $v 4$, available at $\{\mathrm{http}: / / \mathrm{https} / /$ esamultimedia.esa.int/docs/ EarthObservation/CIMR-MRD-v4.0-20201006_Issued.pdf $\}$.

[3] J. Maslanik and J. Stroeve, "DMSP SSM/I-SSMIS Daily Polar Gridded Brightness Temperatures, Version 4," 2004.

[4] T. Markus and D. J. Cavalieri, "An enhancement of the NASA Team sea ice algorithm," IEEE Transactions on Geoscience and Remote Sensing, vol. 38 , no. 3, pp. 1387-1398, may 2000.

[5] J. C. Comiso and F. Nishio, "Trends in the sea ice cover using enhanced and compatible AMSR-E, SSM/I, and SMMR data," Journal of Geophysical Research: Oceans, vol. 113, no. C2, 2008.

[6] G. E. Backus and J. F. Gilbert, "Numerical Applications of a Formalism for Geophysical Inverse Problems," Geophysical Journal International, vol. 13, no. 1-3, pp. 247-276, jul 1967.

[7] A. Stogryn, "Estimates of brightness temperatures from scanning radiometer data," IEEE Transactions on Antennas and Propagation, vol. 26, no. 5, pp. 720-726, 1978.

[8] G. Poe, "Optimum interpolation of imaging microwave radiometer data," IEEE Transactions on Geoscience and Remote Sensing, vol. 28, no. 5, pp. 800-810, 1990.

[9] R. H. Chan and M. K. Ng, "Conjugate gradient methods for Toeplitz systems," SIAM review, vol. 38, no. 3, pp. 427-482, 1996.

[10] D. G. Long and D. L. Daum, "Spatial resolution enhancement of SSM/I data," IEEE Transactions on Geoscience and Remote Sensing, vol. 36, no. 2, pp. 407-417, mar 1998.

[11] D. G. Long, M. J. Brodzik, and M. A. Hardman, "Enhanced-Resolution SMAP Brightness Temperature Image Products," IEEE Transactions on Geoscience and Remote Sensing, vol. 57, no. 7, pp. 4151-4163, jul 2019. 

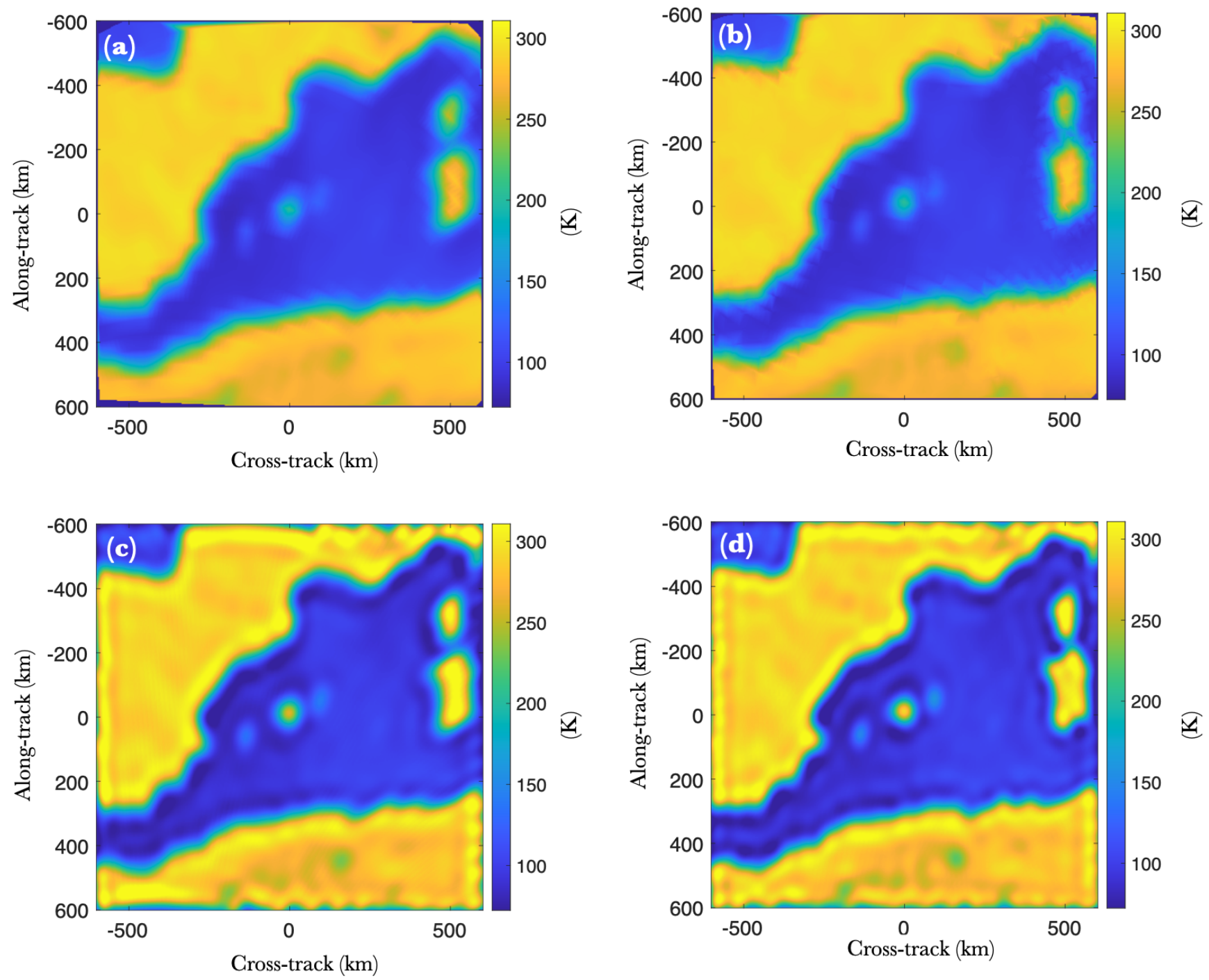

Fig. 10. Noisy CIMR measurements (interpolated onto the finer spatial grid) related to the reference field of Fig. 3c and obtained using FWD scans only (a) and the combination of FWD and FWD+BWD scans (b). Reconstructed fields at enhanced spatial resolution obtained using FWD scans only (c) and the combination of FWD and FWD+BWD scans (d).

[12] F. Lenti, F. Nunziata, M. Migliaccio, and G. Rodriguez, "TwoDimensional TSVD to Enhance the Spatial Resolution of Radiometer Data," IEEE Transactions on Geoscience and Remote Sensing, vol. 52, no. 5, pp. 2450-2458, 2014.

[13] F. Lenti, F. Nunziata, C. Estatico, and M. Migliaccio, "Spatial Resolution Enhancement of Earth Observation Products Using an Acceleration Technique for Iterative Methods," IEEE Geoscience and Remote Sensing Letters, vol. 12, no. 2, pp. 269-273, feb 2015.

[14] M. Migliaccio and A. Gambardella, "Microwave radiometer spatial resolution enhancement," IEEE Transactions on Geoscience and Remote, Sensing, vol. 43, 2005.

[15] A. Gambardella and M. Migliaccio, "On the Superresolution of Microwave Scanning Radiometer Measurements," IEEE Geoscience and Remote Sensing Letters, vol. 5, no. 4, pp. 796-800, oct 2008.

[16] F. Lenti, F. Nunziata, C. Estatico, and M. Migliaccio, "On the Spatial Resolution Enhancement of Microwave Radiometer Data in Banach Spaces," IEEE Transactions on Geoscience and Remote Sensing, vol. 52, no. 3, pp. 1834-1842, mar 2014.

[17] F. Lenti, F. Nunziata, C. Estatico, and M. Migliaccio, "Conjugate Gradient Method in Hilbert and Banach Spaces to Enhance the Spatial Resolution of Radiometer Data," IEEE Transactions on Geoscience and Remote Sensing, vol. 54, no. 1, pp. 397-406, 2016.

[18] F. Lenti, F. Nunziata, C. Estatico, and M. Migliaccio, "Analysis of Reconstructions Obtained Solving lp-Penalized Minimization Problems," IEEE Transactions on Geoscience and Remote Sensing, vol. 53, no. 9, pp. 4876-4886, sep 2015.

[19] M. Alparone, F. Nunziata, C. Estatico, F. Lenti, and M. Migliaccio, "An Adaptive Lp-Penalization Method to Enhance the Spatial Resolution of Microwave Radiometer Measurements," IEEE Transactions on Geoscience and Remote Sensing, pp. 1-10, 2019.

[20] M. Alparone, F. Nunziata, C. Estatico, and M. Migliaccio, "A Multichannel Data Fusion Method to Enhance the Spatial Resolution of Microwave Radiometer Measurements," IEEE Transactions on Geoscience and Remote Sensing, pp. 1-9, 2020.

[21] E. Santi, "An application of the SFIM technique to enhance the spatial resolution of spaceborne microwave radiometers," International Journal of Remote Sensing, vol. 31, no. 9, pp. 2419-2428, 2010.

[22] R. Parinussa, M. Yilmaz, M. Anderson, C. Hain, and R. de Jeu, "An intercomparison of remotely sensed soil moisture products at various spatial scales over the Iberian Peninsula," Hydrological Processes, vol. 28, 2014.

[23] M. Piles, A. Camps, M. Vall-llossera, and M. Talone, "SpatialResolution Enhancement of SMOS Data: A Deconvolution-Based Approach," IEEE Transactions on Geoscience and Remote Sensing, vol. 47, no. 7, pp. 2182-2192, jul 2009.

[24] G. Portal, M. Vall-llossera, M. Piles, A. Camps, D. Chaparro, M. Pablos, and L. Rossato, "A Spatially Consistent Downscaling Approach for SMOS Using an Adaptive Moving Window," IEEE Journal of Selected Topics in Applied Earth Observations and Remote Sensing, vol. 11, no. 6 , pp. $1883-1894$, jun 2018 . 

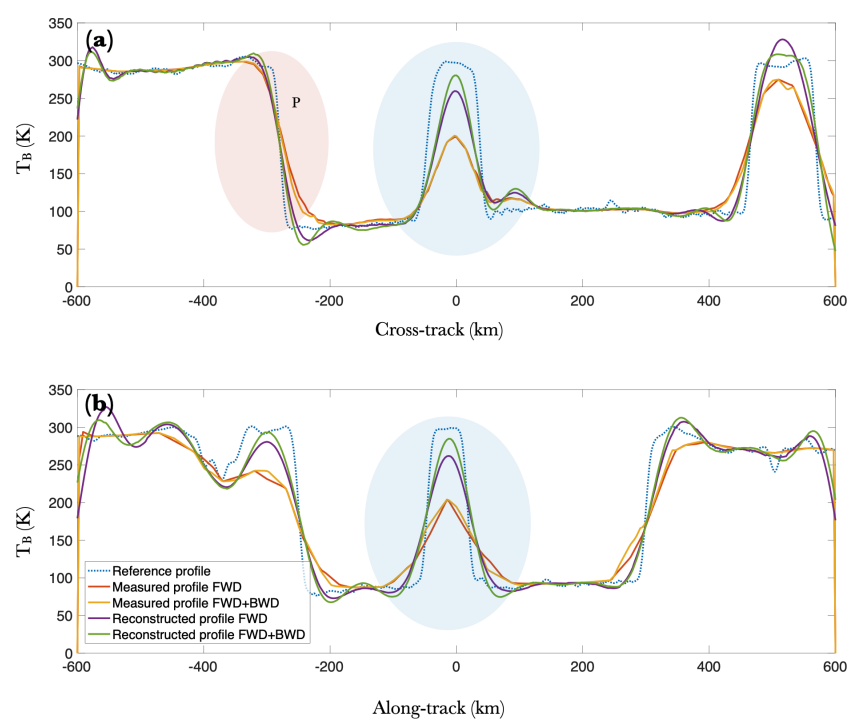

Fig. 11. Brightness temperature profiles related to the along-scan (a) and across-scan (b) transects depicted in Fig. 3c evaluated using the field reconstructed at enhanced spatial resolution and the measured field obtained using the FWD only and the FWD+BWD combination. Note that the reference profile is also annotated.
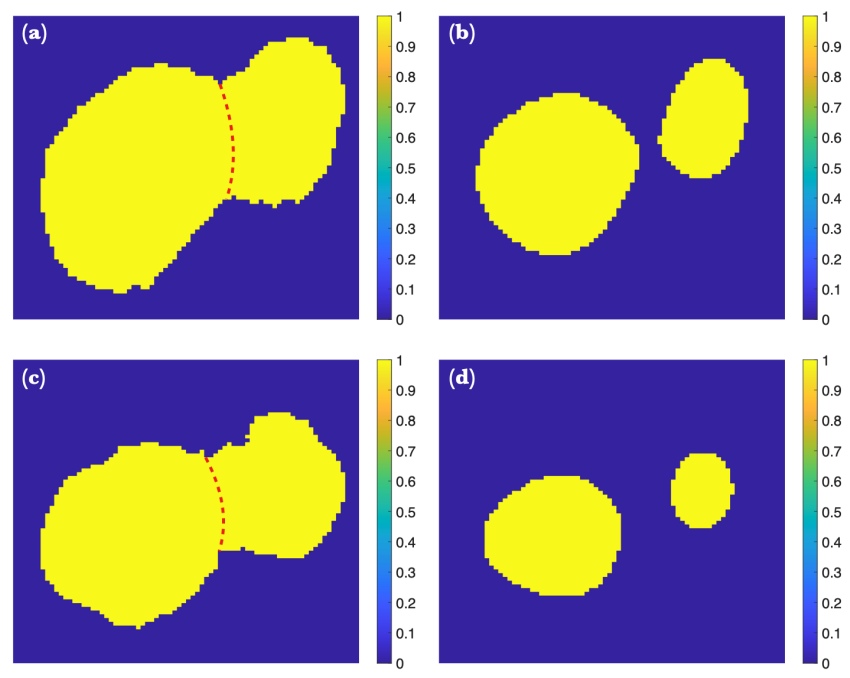

Fig. 12. $-3 \mathrm{~dB}$ binary masks obtained from zoomed-in sections of the fields over the island of Mallorca, after normalising the brightness temperature to its maximum and adopting a $\mathrm{dB}$ scale. The image is arranged in matrix format where the rows stands for the FWD scans only (first row) and FWD+BWD scans (second row) while the columns stands for the CIMR measurements interpolated onto the finer spatial grid (first column) and the reconstructed fields at enhanced spatial resolution (second column). The red dotted line delimits the area used to evaluate the IF metric over the Mallorca island

[25] H. Song, G. Wang, A. Cao, Q. Liu, and B. Huang, "Improving the Spatial Resolution of FY-3 Microwave Radiation Imager via Fusion With FY-3/MERSI," IEEE Journal of Selected Topics in Applied Earth Observations and Remote Sensing, vol. 10, no. 7, pp. 3055-3063, 2017.

[26] M. J. Brodzik, B. Billingsley, T. Haran, B. Raup, and M. H. Savoie, "EASE-Grid 2.0: Incremental but Significant Improvements for EarthGridded Data Sets," ISPRS International Journal of Geo-Information, vol. 1, no. 1, pp. 32-45, mar 2012.

[27] M. Brodzik, B. Billingsley, T. Haran, B. Raup, and M. Savoie, "Correction: Brodzik, M.J., et al. EASE-Grid 2.0: Incremental but Significant Improvements for Earth-Gridded Data Sets. ISPRS International Journal of Geo-Information 2012, 1, 32-45," ISPRS International Journal of
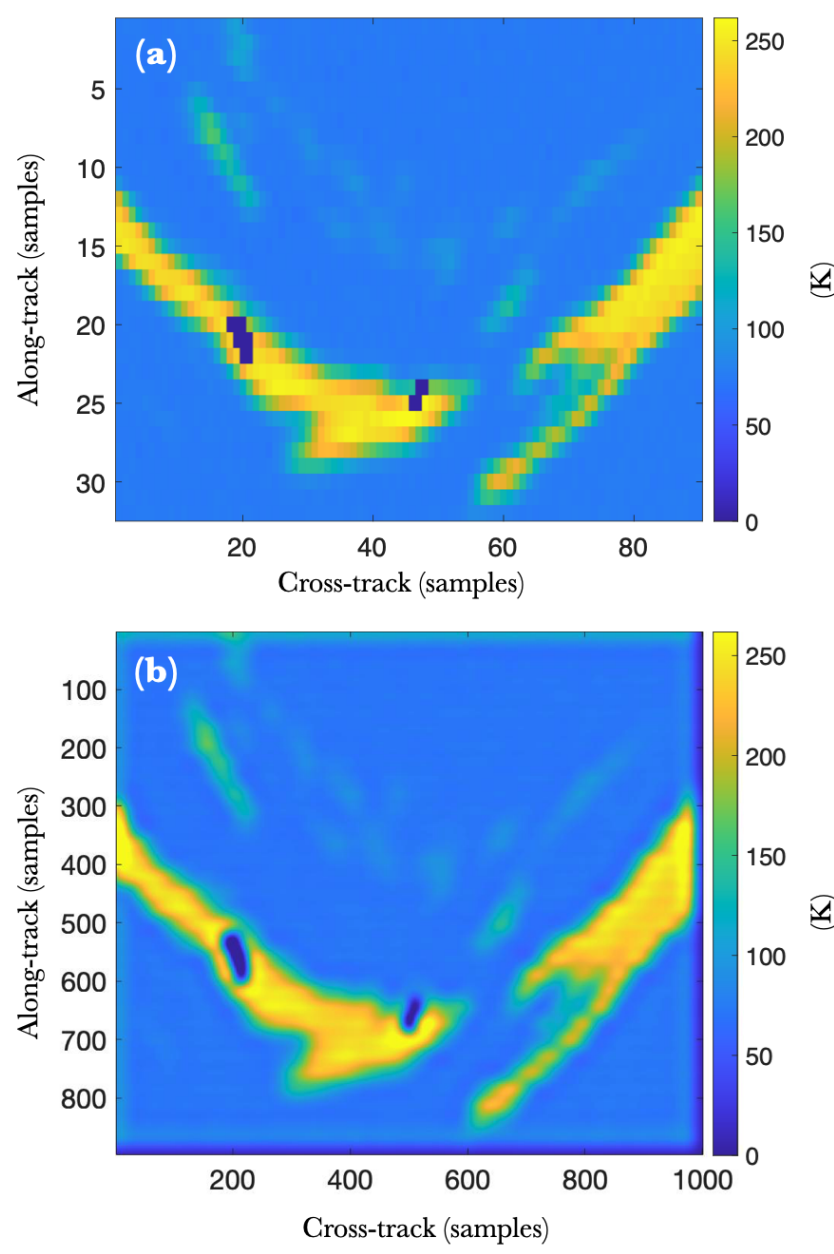

Fig. 13. (a) SMAP L-band radiometer measurement collected in 2021 over portions of Cuba, Haiti and the Bahamas archipelago; (b) Reconstruction obtained using LW. Note that black pixels stand for gaps values that can occur under the conditions explained in the data User Guide [32]

Geo-Information, vol. 3, no. 3, pp. 1154-1156, sep 2014.

[28] F. Schöpfer, A. K. Louis, and T. Schuster, "Nonlinear iterative methods for linear ill-posed problems in Banach spaces," Inverse Problems, vol. 22, no. 1, pp. 311-329, feb 2006.

[29] A. Quarteroni, R. Sacco, and F. Saleri, Numerical Mathematics, ser. Texts in applied mathematics. Springer, 2000.

[30] TICRA, GRASP Software, available at https://www.ticra.com/software/ grasp/.

[31] D. Entekhabi, E. G. Njoku, P. E. O'Neill, K. H. Kellogg, W. T. Crow, W. N. Edelstein, J. K. Entin, S. D. Goodman, T. J. Jackson, J. Johnson, J. Kimball, J. R. Piepmeier, R. D. Koster, N. Martin, K. C. McDonald, M. Moghaddam, S. Moran, R. Reichle, J. C. Shi, M. W. Spencer, S. W. Thurman, L. Tsang, and J. V. Zyl, "The Soil Moisture Active Passive (SMAP) Mission," Proceedings of the IEEE, vol. 98, no. 5, pp. 704-716, 2010.

[32] J. R. Piepmeier, P. Mohammed, J. Peng, E. J. Kim, G. D. Amici, J. Chaubell, and C. Ruf, SMAP L1B Radiometer Half-Orbit TimeOrdered Brightness Temperatures, Version 5, available at https://doi.org/ 10.5067/ZHHBN1KQLI20. 